\title{
Far-infrared elastic scattering proposal for the Avogadro Project's silicon spheres
}

\author{
Muhammad Hamza Humayun a,b,1, Imran Khan ${ }^{a, c}$, Farhan Azeem ${ }^{a}$, \\ Muhammad Rehan Chaudhry a, Ulaş Sabahattin Gökay a,d,2, Mohammed Sharif Murib a,e,3, \\ Ali Serpengüzel ${ }^{\mathrm{a}, 4, *}$ \\ ${ }^{a}$ Koç University, Microphotonics Research Laboratory, Department of Physics, Rumelifeneri Yolu, Sariyer, Istanbul 34450 Turkey \\ ${ }^{\mathrm{b}}$ Bilkent University, Department of Electrical and Electronics Engineering, Bilkent, Ankara 06800 Turkey \\ c INFN, Sezione di Roma Tor Vergata, Roma I-00133 Italy and Gran Sasso Science Institute, L'Aquila I-67100 Italy \\ d Işlk University/OPAM, Department of Mechanical Engineering, Meșrutiyet Mahallesi, Şile, Istanbul 34980 Turkey \\ e Gent University/IMEC, Department of Information Technology, INTEC Technologiepark, Zwijnaarde 15, Gent B-9052 Belgium
}

\section{A R T I C L E I N F O}

Article history:

Received 4 August 2017

Revised 23 November 2017

Accepted 22 December 2017

Available online 27 December 2017

\section{Keywords:}

Avogadro constant

Avogadro Project

Elastic scattering

Far-infrared spectroscopy

Lorenz-Mie theory

Morphology-dependent resonances

Near-infrared spectroscopy

Optical interferometry

Silicon sphere

Terahertz spectroscopy

Whispering gallery modes

\begin{abstract}
A B S T R A C T
Avogadro constant determines the number of particles in one mole of a substance, thus relating the molar mass of the substance to the mass of this substance. Avogadro constant is related to Système Internationale base units by defining the very concept of chemical quantity. Revisions of the base units created a need to redefine the Avogadro constant, where a collaborative work called the Avogadro Project is established to employ optical interferometry to measure the diameter of high quality $100 \mathrm{~mm}$ silicon spheres. We propose far-infrared spectroscopy for determining the Avogadro constant by using elastic scattering from the $100 \mathrm{~mm}$ Avogadro Project silicon spheres. Similar spectroscopic methods are already in use in the near-infrared, relating whispering gallery modes of the $1 \mathrm{~mm}$ silicon spheres to the diameter of the spheres. We present numerical simulations in the far-infrared and the near-infrared, as well as spatially scaled down elastic scattering measurements in the near-infrared. These numerical and experimental results show that, the diameter measurements of $100 \mathrm{~mm}$ single crystal silicon spheres with elastic scattering in the far-infrared can be considered as an alternative to optical interferometry.
\end{abstract}

(c) 2017 Elsevier Ltd. All rights reserved.

\section{Introduction}

The kilogram is the Système Internationale (SI) unit of mass, which is defined with the mass of a material artifact kept at Bureau International des Poids et des Measures (BIPM) [1,2], where Avogadro constant $\left(\mathrm{N}_{\mathrm{A}}\right)$ relates the number of atoms in the artefact to the molar mass of the material. However, for the definition of a base unit, it is imperative that, the defining quantity does not experience a change in its physical state with the passage of time. Thus, a need of redefinition for the kilogram standard has arisen [3]. A global effort has been ongoing to redefine the mass of the prototype in terms of fundamental constants or the mass

\footnotetext{
* Corresponding author.

E-mail address: aserpenguzel@ku.edu.tr (A. Serpengüzel).

1 https://orcid.org/0000-0002-2908-5154

2 https://orcid.org/0000-0003-3785-2285

3 https://orcid.org/0000-0002-8761-4253

4 https://orcid.org/0000-0002-0676-8817
}

of an atom, which can have small enough uncertainty to replace the current definition of the kilogram [4]. In this case, recalculating $\mathrm{N}_{\mathrm{A}}$ with sufficient accuracy became the focus. In the past 40 years, using interferometric methods to measure the diameter of an extremely well-shaped and pure silicon (Si) sphere with low uncertainty became a fundamental approach [5].

The use of optical interferometry for the diameter measurement of spheres was first setup by Saunders [6]. Over the years, the improvement and the novelty in interferometry resulted in more precise and accurate measurements. The material choice for the sphere was $\mathrm{Si}$, where increasing production purity of the Si spheres helped the researchers. Diameter measurements for Si spheres by using interference yielded an uncertainty on the orders of $10^{-8}$ $[7,8]$, and use of different interferometry methods such as scanning type and Fizeau interferometer helped improve the measurement conditions [9-12]. Recently, laser interferometry with a flat etalon on two spheres [13] combined with the modern mathematical methods [14] helped bringing down the uncertainty in diameter measurements to less than $1 \mathrm{~nm}$. Recently, relative standard 
uncertainty in these spheres is reported to be on the order of $2.2 \times 10^{-8}[15]$.

Avogadro spheres, with their $100 \mathrm{~mm}$ diameters, are macroscopic objects. On the microscopic scale, Si microspheres [16] provide confinement of light in all three dimensions (3D), and can act as optical fibre coupled [17] 3D optical microcavities with high quality factor (Q-factor) whispering gallery modes (WGMs), which sensitively depend on the diameter of the sphere [18-20]. Size measurement by using light scattering for microparticles is wellknown [21-23]. Dielectric spheres with dimensions much larger than the wavelength of light can sustain high Q-factor WGMs [24]. Schiller et al. performed resonance experiments on a $38 \mathrm{~mm}$ diameter fused-silica sphere [25].

Additionally, far-IR time domain WGM spectroscopy of a $\mathrm{Si}$ cylinder with a diameter of $5 \mathrm{~mm}$ and excited by a Si waveguide with a thickness of $100 \mu \mathrm{m}$ at $1 \mathrm{THz}$ [26] has been reported. Here we propose, for the first time to the best of our knowledge, farIR elastic scattering WGM spectroscopy to measure the diameter of the Avogadro Project Si spheres. Our proposal offers the benefit of using macroscopic Si spheres with the excitation of their WGMs to measure their diameter with far-IR spectroscopy. We structure the paper as follows. We demonstrate our approach experimentally with the elastic scattering measurements from a $1 \mathrm{~mm}$ single crystal Si sphere in the near-IR. We perform Gaussian beam elastic scattering simulations for the $1 \mathrm{~mm}$ Si sphere and for the $100 \mathrm{~mm}$ Avogadro sphere. Finally, we propose a far-IR resonance spectroscopy setup for the elastic scattering diameter measurements for the $100 \mathrm{~mm}$ Avogadro sphere.

\section{Avogadro Project}

The Avogadro Project proposes to redefine the kilogram in terms of one of the Avogadro constant $\left(\mathrm{N}_{\mathrm{A}}\right)$ as [27]:

$1 \mathrm{~kg}=\left\{\mathrm{N}_{A}\right\} \frac{m\left({ }^{12} \mathrm{C}\right)}{0.012}$

where $\mathrm{m}\left({ }^{12} \mathrm{C}\right)$ is the molar mass of carbon. The conditions for redefinition of the kilogram have been formulated, and a relative uncertainty of 2 parts in $10^{8}$ is required for redefinition of unit of mass [28]. For finding $N_{A}$, a single crystal ${ }^{28} \mathrm{Si}$ isotope is used [29]. $\mathrm{Si}$ crystal was chosen due to the presence of already mature semiconductor industry based on Si with well-known mechanical, thermal, electrical, and chemical properties, so that, highly pure and almost perfect Si spheres can be manufactured [30,31]. Counting the atoms in a single crystal $\mathrm{Si}$ sphere for determining $\mathrm{N}_{\mathrm{A}}$ for redefinition of the mass unit was previously outlined [32]. X-ray crystal density molar mass (XRCDMM) method can be used to find $\mathrm{N}_{\mathrm{A}}$ by utilizing the macroscopic density $\rho$ of the ideal Si crystal, the molar mass $\mathrm{m}\left({ }^{28} \mathrm{Si}\right)$ of $\mathrm{Si}$, and the unit cell volume $\mathbf{a}^{\mathbf{3}}$ of the $\mathrm{Si}$ crystal as follows $[33,34]$ :

$\mathrm{N}_{\mathrm{A}}=\mathrm{nV}_{\mathrm{mol}} / \mathbf{a}^{3}$

$\mathrm{V}_{\mathrm{mol}}=\mathrm{m}\left({ }^{28} \mathrm{Si}\right) / \rho$

where $\mathrm{V}_{\mathrm{mol}}$ is the molar volume of $\mathrm{Si}$, and $\mathrm{n}$ the number of atoms in the unit cell. Thus, XRCDMM method facilitates the determination of $\mathrm{N}_{\mathrm{A}}$ in terms of macroscopic parameters instead of counting a large number of atoms with a very small uncertainty. The density of the Si can be found by dividing the mass of the sphere $\mathrm{M}$ by the volume $\mathrm{V}$ as,

$\rho=\mathrm{M} / \mathrm{V}$

The volume of the Si sphere can be found by measuring its diameter. Uncertainty in the diameter measurement decides the uncertainty in the volume calculations. To achieve an accuracy of $0.01 \mathrm{ppm}$ in volume, the diameter must be known to a range

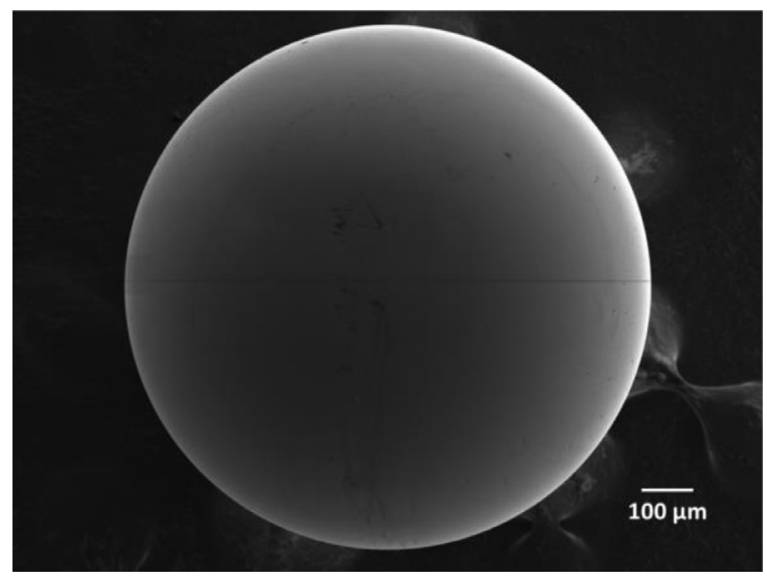

Fig. 1. Scanning electron microgram (SEM) of the $1 \mathrm{~mm}$ diameter silicon sphere.

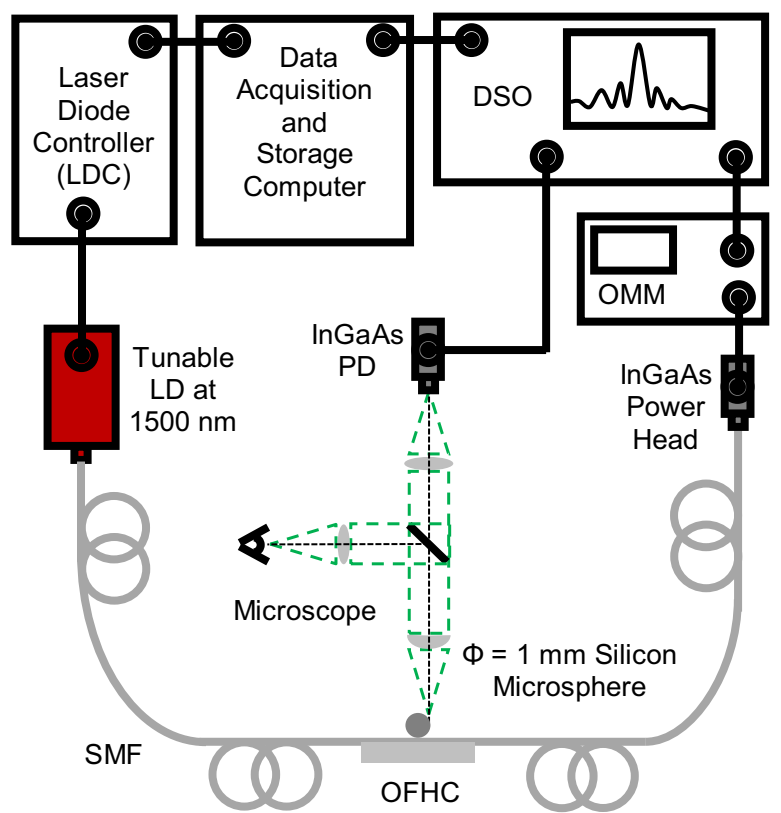

Fig. 2. Experimental setup for near-IR $90^{\circ}$ elastic scattering and $0^{\circ}$ transmission spectroscopy of the $1 \mathrm{~mm}$ Si sphere.

of $0.6 \mathrm{~nm}$, i.e., within one atomic spacing. In diameter measurements the effect of the surface native and non-native oxides (with a thickness on the order of $1.07 \mathrm{~nm}$ ) has a relative uncertainty of $3.4 \times 10^{-8}[35]$.

\section{Near-IR elastic scattering of the $\mathbf{1} \mathbf{~ m m}$ silicon sphere}

Fig. 1 shows a scanning electron microgram (SEM) of the $1 \mathrm{~mm}$ diameter Si sphere (Ball Semiconductor). The $1 \mathrm{~mm}$ Si sphere was produced by the solidification of melted droplets of $\mathrm{Si}$ falling through a tube. After the rapid solidification, single crystal silicon spheres are evidences by the formation of 8 horns in the 111 crystal directions. These horns are attributed to the volume expansion of Si from liquid to solid phase, and the difference of the solidification speed in crystal orientation, as the 111 direction has the slowest solidification speed, the excess liquid is maintained in 111 direction, until the end of the solidification with the formation of the horns. Later, the Si beads were lapped and polished mechanically to make a mirror-like finish with no surface defects and damages [36,37].

Fig. 2 shows our near-IR WGM excitation setup. Tunable distributed feedback (DFB) diode lasers (operating at 1428 and 


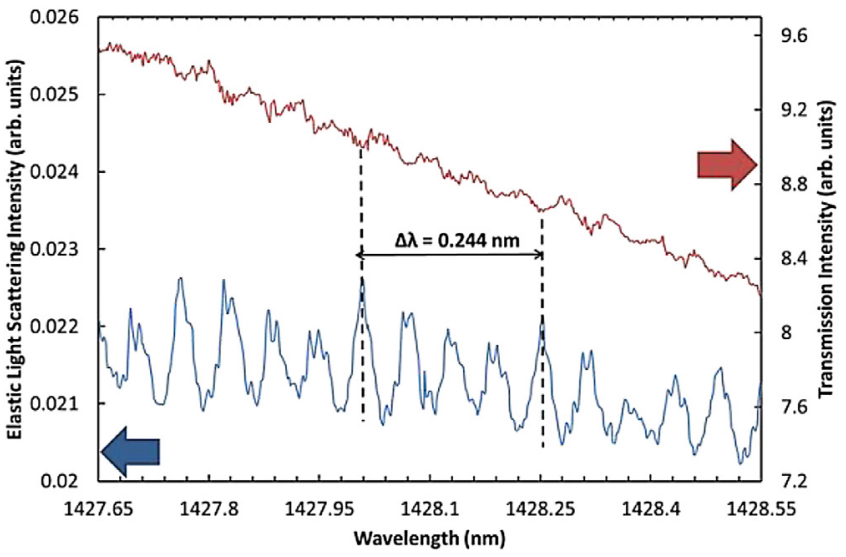

Fig. 3. Near-IR $(1428 \mathrm{~nm}) 90^{\circ}$ elastic scattering and $0^{\circ}$ transmission spectra from the $1 \mathrm{~mm}$ silicon sphere.

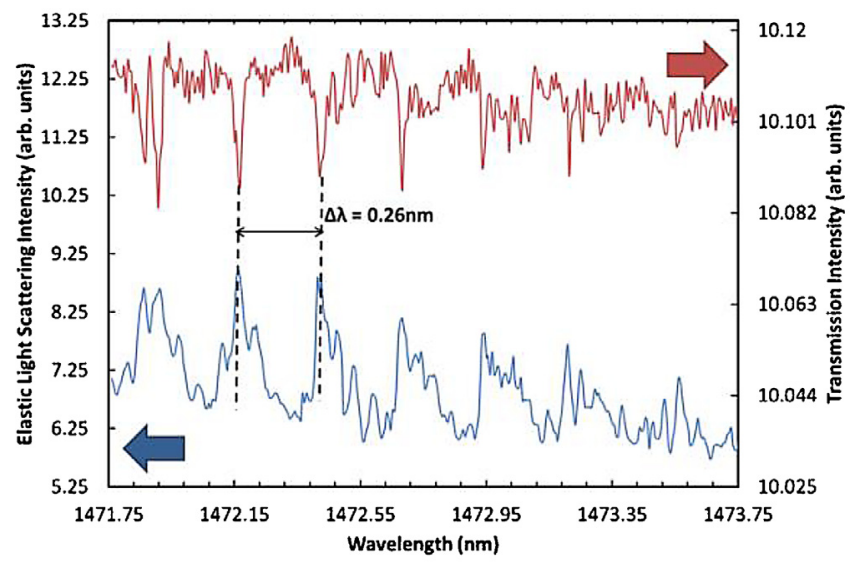

Fig. 4. Near-IR $(1472 \mathrm{~nm}) 90^{\circ}$ elastic scattering and $0^{\circ}$ transmission spectra from the $1 \mathrm{~mm}$ silicon sphere.

$1472 \mathrm{~nm}$ ) are used to excite the WGMs of the Si sphere. The DFB lasers are driven by a laser diode controller (LDC) connected to a computer, where the output wavelength of a DFB laser is controlled by the LabView (National Instruments) software. The DFB laser output is fed to the single mode fibre (SMF), which is connected to the optical fibre half coupler (OFHC). The OFHC is manufactured from polished SMF, which couples $99.4 \%$ of the light passing through the coupler, when an index-matching oil is placed on the OFHC [38]. The SMF cladding thickness on the OFHC surface is on the order of a micrometer.

The Si microsphere is placed on the top of the OFHC, where the light in the SMF is evanescently coupled to the Si microsphere $[39,40]$. An optical microscope collects the elastically scattered light from the $\mathrm{Si}$ microsphere at $90^{\circ}$. This collected light is incident on the InGaAs photodetector (PD), which is connected to a digital storage oscilloscope (DSO). The $0^{\circ}$ transmitted signal is collected by the InGaAs power head (PH) of an optical multimeter (OMM). General purpose interface bus (GPIB) protocol is used to connect the DSO and the OMM to the data acquisition computer.

\section{Near-IR elastic scattering and transmission spectra for the $1 \mathrm{~mm}$ silicon sphere}

Fig. 3 shows the $90^{\circ}$ elastic scattering and the $0^{\circ}$ transmission for near-infrared (near-IR) light (with a central wavelength at $1428 \mathrm{~nm}$ ). Fig. 4 shows the $90^{\circ}$ elastic scattering and $0^{\circ}$ transmission for near-IR light (with central wavelength at $1472 \mathrm{~nm}$ ). The elastic light scattering spectra in Figs. 3 and 4 show the expected dips in the $0^{\circ}$ transmission and the corresponding peaks in the $90^{\circ}$ elastic scattering. In Fig. 3, the experimentally measured mode spacing of the WGMs is $0.244 \mathrm{~nm}$ for the DFB laser at $1428 \mathrm{~nm}$, and correlates well with the calculated mode spacing of $0.249 \mathrm{~nm}$ in the theoretical model. In Fig. 4, the experimentally measured mode spacing of the WGMs is $0.252 \mathrm{~nm}$ for the DFB laser with central wavelength $1472 \mathrm{~nm}$, and correlates well with the $0.265 \mathrm{~nm}$ of the theoretical model. Using Eq. (8), the Q-factor is found to be $6 \times 10^{4}$ for the WGMs of Fig. 3, and $\approx 5 \times 10^{4}$ for the WGMs of Fig. 4 . These relatively broad WGM linewidths can be reduced by several orders of magnitude with improved experimental conditions such as using narrower linewidth lasers.

\section{Comparison of $100 \mathrm{~mm}$ Avogadro Project Si sphere and $1 \mathrm{~mm}$ Si sphere}

The most recent determination of the Avogadro constant, $N_{A}=6.02214076(12) \times 10^{23} \mathrm{~mol}^{-1}$ achieved the target of relative standard uncertainty of $2.0 \times 10^{-8}$. This is currently the most accurate determination of a fundamental constant, which can be used for a new definition of the kilogram. Table 1 contains the $\mathrm{x}$ ray crystal density (XRCD) method results of this recent work. In Table 1, we also compare the properties of the $100 \mathrm{~mm}$ Avogadro sphere and the $1 \mathrm{~mm}$ Si sphere.

By taking the refractive index of $\mathrm{Si}$ at $1500 \mathrm{~nm}$ as 3.48 [41], and using Eqs. (6) and (7) on Fig. 3 results, the diameter of the $1 \mathrm{~mm}$ sphere was calculated to be $1022 \mu \mathrm{m}$. From the SEM (ZEISS Ultra plus field emission SEM) image of our $1 \mathrm{~mm} \mathrm{Si} \mathrm{sphere} \mathrm{(Fig.} \mathrm{1),} \mathrm{the}$ diameter was found to be $1017 \mu \mathrm{m}$, agreeing to within $0.5 \%$ with our spectral measurement. Additionally, by taking the values of the molar mass $m\left({ }^{28} \mathrm{Si}\right)$, the lattice constant, and the $\mathrm{N}_{\mathrm{A}}$ from CODATA [42], the mass of the $1 \mathrm{~mm}$ sphere was calculated to be $1.222 \mathrm{mg}$ (Table 1). The mass (Mettler Toledo Excellence Plus XP6 Micro Balance) of the $1 \mathrm{~mm}$ sphere was found to be $1.257 \mathrm{mg}$ agreeing with the calculated value to within $3 \%$.

If the size obtained is the correct one, it should be correct, when measured in different spectral regions, i.e., near IR, mid-IR, far-IR, or in the same spectral region at different spectral bands, different thicknesses of the cladding on the SMF, different positions of the collection optics, and different polarizations of the excitation laser. All these potential multiplexed spectroscopic measurements will contribute to the precision of the measurement of the diameter of the Avogadro sphere.

In addition, the sphericity measurements can be performed by rotating the sphere in various directions and acquiring the respective spectra. If an excitation laser close to the absorption bands of silicon is used, it would be possible to heat up the silicon sphere, and study the thermal tuning of the elastic scattering spectra.

\section{GLMT simulations for the $1 \mathrm{~mm} \mathrm{Si}$ sphere and the $100 \mathrm{~mm}$ Avogadro Project Si sphere}

Our numerical simulations of the $1 \mathrm{~mm} \mathrm{Si}$ sphere and the $100 \mathrm{~mm}$ Avogadro sphere uses Lorenz-Mie theory [43]. An incident electromagnetic wave can be trapped inside the surface of a dielectric sphere with the help of total internal reflection (TIR) and result in WGMs [44]. WGMs manifest themselves as peaks in the elastic scattering spectrum as a function of the size parameter:

$x=\frac{\pi \phi \mu_{0}}{\lambda}$

where $\phi$ is the diameter of dielectric sphere, $\lambda$ the vacuum wavelength of light, and $\mu_{0}$ the refractive index of the surrounding medium. The variation of the wavelength as a function of the vari- 
Table 1

Experimental parameter comparison of the $100 \mathrm{~mm}$ far-infrared Avogadro sphere and the $1 \mathrm{~mm}$ near-infrared silicon sphere.

\begin{tabular}{|c|c|c|c|c|c|}
\hline parameter & symbol & $100 \mathrm{~mm}$ silicon sphere & reference & $1 \mathrm{~mm}$ silicon sphere & reference \\
\hline silicon molar mass $\left(\mathrm{g} \mathrm{mol}^{-1}\right)$ & $\mathrm{m}\left({ }^{28} \mathrm{Si}\right)$ & $27.97697009(15)$ & 15 & $27.97697009(15)$ & 42 \\
\hline silicon atoms per unit cell & $\mathrm{n}$ & 8 & 15 & 8 & 42 \\
\hline silicon lattice constant (pm) & $\mathrm{a}$ & $543.0996219(10)$ & 15 & $543.1020504(89)$ & 42 \\
\hline silicon density $\left(\mathrm{kg} \cdot \mathrm{m}^{-3}\right)$ & $\rho$ & $2320.070943(46)$ & 15 & $2320.070959(49)$ & 42 \\
\hline Avogadro constant $\left(10^{23} \mathrm{~mol}^{-1}\right)$ & $\mathrm{N}_{\mathrm{A}}$ & $6.02214072(13)$ & 15 & $6.02214082(18)$ & 42 \\
\hline standard uncertainty $\left(10^{23} \mathrm{~mol}^{-1}\right)$ & $\delta \mathrm{N}_{\mathrm{A}}$ & $2.0 \times 10^{-8}$ & 15 & $3.0 \times 10^{-8}$ & 42 \\
\hline silicon sphere diameter $(\mathrm{mm})$ & $\phi$ & $93.71081111(62)$ & 15 & $1.020(03)$ & this work \\
\hline silicon sphere volume $\left(\mathrm{cm}^{3}\right)$ & V & $430.8912891(69)$ & 15 & $0.0044451776(09)$ & this work \\
\hline silicon sphere mass $(\mathrm{g})$ & M & $999.698359(11)$ & 15 & $0.001222(05)$ & this work \\
\hline silicon resistivity $(\mathrm{k} \Omega \mathrm{cm})$ & $\sigma^{-1}$ & 10 & 51 & 10 & 53 \\
\hline silicon refractive index & $\mu$ & 3.40 & 57 & 3.48 & 41 \\
\hline silicon absorption coefficient $\left(\mathrm{cm}^{-1}\right)$ & $\alpha$ & 0.02 & 51 & 0.01 & 42 \\
\hline silicon sphere quality factor & Q & $10^{8}$ & proposed work & $5 \times 10^{4}$ & this work \\
\hline excitation wavelength (nm) & $\lambda$ & 150,000 & proposed work & $1500(50)$ & this work \\
\hline excitation frequency $(\mathrm{THz})$ & $v$ & 2 & proposed work & $200(07)$ & this work \\
\hline silicon sphere size parameter & $\mathrm{x}$ & 2094 & proposed work & 2094 & this work \\
\hline silicon sphere WGM spacing (nm) & $\Delta \lambda$ & 25.5 & proposed work & $0.253(11)$ & this work \\
\hline
\end{tabular}

ation of the size parameter $x$ is given as:

$\frac{\Delta \lambda}{\lambda}=\frac{\Delta x}{x}$

The mode spacing $\Delta x$ between two WGMs, with consecutive polar angular mode numbers and with the same radial mode order as a function of size parameter $x$, can be calculated as [45]:

$\Delta x=\frac{\tan ^{-1} \sqrt{m^{2}-1}}{\sqrt{m^{2}-1}}$

where $m=\mu_{/} \mu_{o}$ is the relative refractive index ratio, and $\mu$ is the refractive index of the sphere. We have to keep in mind that, Eq. (6) is an estimate to the overall mode spacing, and more accurate determination of the Avogadro Number can be obtained by fitting the measured spectra for different combinations of mode numbers. Then, Eqs. (4)-(6) can be used to find the diameter of dielectric sphere as [46]:

$\phi=\left[\frac{\lambda^{2}}{\Delta \lambda}\right]\left[\frac{\Delta x}{\pi}\right]=\left[\frac{\lambda^{2}}{\Delta \lambda}\right]\left[\frac{\tan ^{-1} \sqrt{m^{2}-1}}{\pi \sqrt{m^{2}-1}}\right]$

One of the main parameters of the spherical resonator is the Qfactor of the WGMs, which is a measure of the sensitivity of the resonator. The Q-factor is spectrally defined as:

$Q=\frac{\lambda_{R}}{\delta \lambda}$

where $\lambda_{R}$ is the resonant wavelength, and $\delta \lambda$ the linewidth of the resonance peak [47]. For high precision measurements, high Q-factor Si spherical resonators, with minimum optical losses due to absorption and scattering, are desirable. High Q-factors can be obtained, if the sphere is protected from the atmosphere, and the losses are controlled during the manufacturing and the storage stage. The limit for achieving high "material Q-factor" is defined by [48]:

$Q=\frac{2 \pi \mu}{\lambda \alpha}$

where $\alpha$ the absorption coefficient of material. The highest $\mathrm{Q}$ recorded for $\mathrm{Si}$ is on the order of $10^{6}$ for planar $\mathrm{Si}$ microdisk resonators [49], and $10^{8}$ for chemically etched wedge-resonators on Si chip [50].

The quality factor of the WGMs for the Si spheres should be affected with non-negligible absorption of the Si. This intrinsic material loss should limit the unloaded quality factor. The lowest absorption coefficient of the Si reported at $150 \mu \mathrm{m}$ is around $0.02 \mathrm{~cm}^{-1}$ at room temperature for Si resistivity more than $10 \mathrm{k} \Omega \mathrm{cm}$ [51]. This absorption coefficient is on the same order of magnitude as the absorption coefficient of Si, i.e., $0.01 \mathrm{~cm}^{-1}$ [52] in near-IR at $1.5 \mu \mathrm{m}$ at room temperature for Si resistivity on the order of $10 \mathrm{k} \Omega \mathrm{cm}$ [53].

Elastic scattering simulations were performed using a Gaussian beam excitation algorithm [54] of generalized Lorenz-Mie theory (GLMT) with transverse electric (TE) and transverse magnetic (TM) polarization incident beams on the $1 \mathrm{~mm}$ Si sphere and the $100 \mathrm{~mm}$ Avogadro sphere. The half-width-at-half-maximum (HWHM) of the Gaussian beam $\omega_{0}$, was chosen as $56 \mu \mathrm{m}$, and the impact parameter $\mathrm{b}$ as $556 \mu \mathrm{m}$ for the $1 \mathrm{~mm}$ sphere. The impact parameter $b$ defines the vertical distance of the microsphere centre to the excitation source as defined by [55]:

$b=(2 n+1) \frac{\phi}{x}$

The impact parameter associates the angular momentum quantum number $\mathrm{n}$ of the excited WGMs to the lateral distance of the beam to the centre of the sphere through the localization principle [56].

The HWHM of the Gaussian beam $\omega_{0}$, was chosen as $5.513 \mathrm{~mm}$ and impact parameter $b$ as $55.513 \mathrm{~mm}$ for the $100 \mathrm{~mm}$ Si sphere. The rather high HWHM values were chosen due to computational limitations. To assure SMF excitation, these values of $\omega_{0}$ should be reduced by an order of magnitude. The far-IR wavelength of operation is chosen as $150 \mu \mathrm{m}(2 \mathrm{THz})$, i.e., 100 times greater than the near-IR wavelength of $1.5 \mu \mathrm{m}(200 \mathrm{THz})$. The refractive index at $150 \mu \mathrm{m}$ is taken as 3.4 [57].

Figs. 5-8 show the GLMT elastic scattering simulations for transverse electric (TE) and transverse magnetic (TM) polarizations for the $1 \mathrm{~mm} \mathrm{Si}$ sphere and the $100 \mathrm{~mm}$ Avogadro sphere. The size parameter was kept at the same value of $\mathrm{x}=2094$ for both $\mathrm{Si}$ spheres. The spectral mode spacing of $0.25 \mathrm{~nm}$ was found for the $1 \mathrm{~mm}$ Si sphere, which correlates well with the experimentally observed value (Figs. 3 and 4). The $25.5 \mathrm{~nm}$ spectral mode spacing found for the $100 \mathrm{~mm}$ Avogadro sphere is 100 times greater, due to the 100 times scaling of the Si sphere sizes and the spectral ranges. Numerically simulated elastic scattering calculations of the Avogadro sphere show promise for the proposed far-IR elastic scattering diameter measurement method.

\section{Proposed far-IR elastic scattering setup for the $100 \mathrm{~mm}$ Avogadro sphere}

Fig. 9 shows our proposed far-IR elastic scattering setup for measuring the diameter of the Avogadro sphere. The tunable laser to be used as the excitation source can operate at a wavelength of $150 \mu \mathrm{m}(2 \mathrm{THz})$, therefore keeping the size parameter of both 


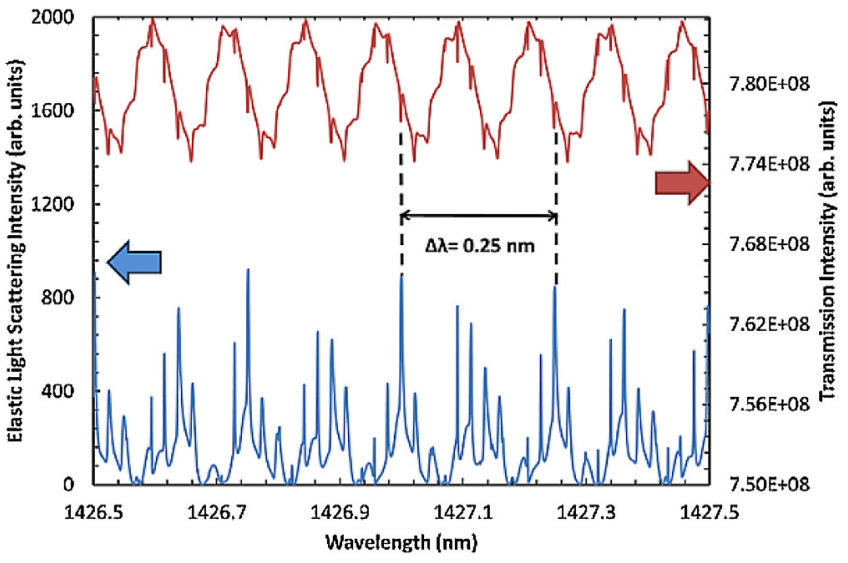

Fig. 5. Near-IR $(1427 \mathrm{~nm}) \mathrm{TE} 90^{\circ}$ elastic scattering and $0^{\circ}$ transmission simulation spectra for the $1 \mathrm{~mm}$ silicon sphere.

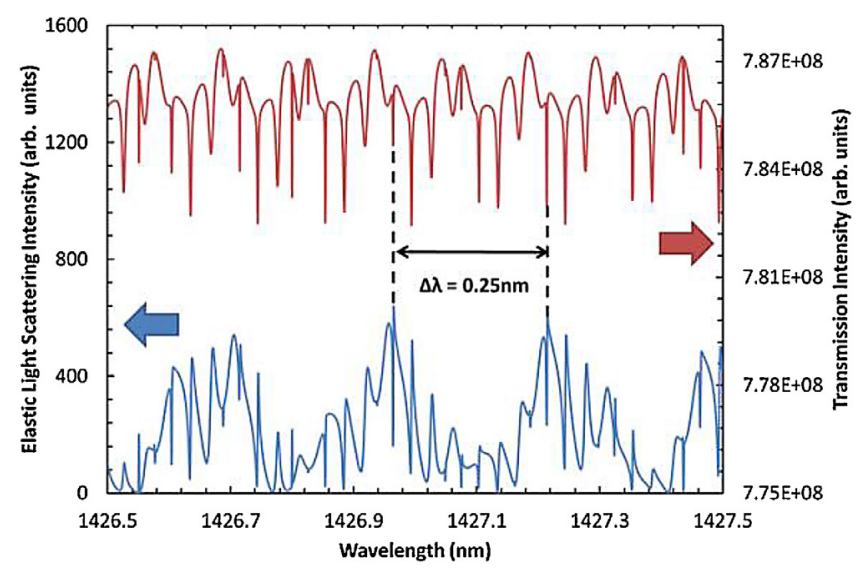

Fig. 6. Near-IR $(1427 \mathrm{~nm}) \mathrm{TM} 90^{\circ}$ elastic scattering and $0^{\circ}$ transmission simulation spectra for the $1 \mathrm{~mm}$ silicon sphere.

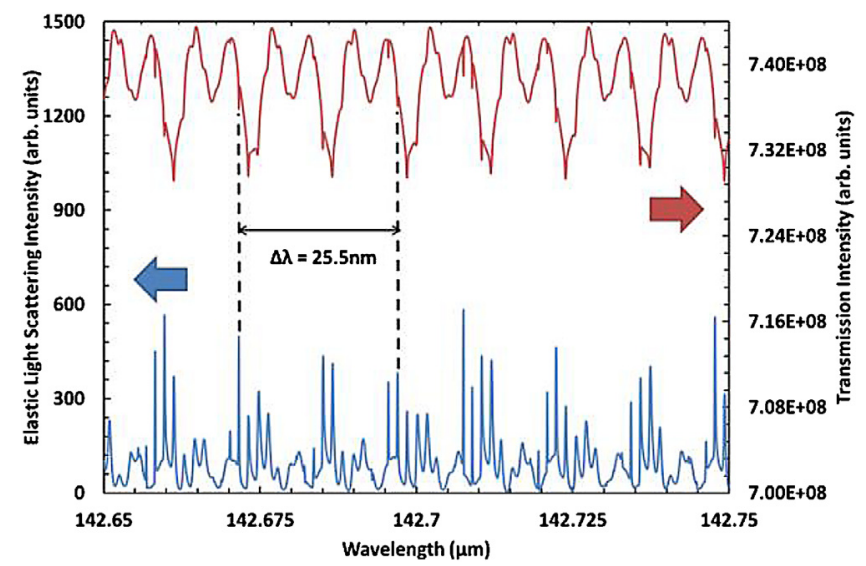

Fig. 7. Far-IR $(142 \mu \mathrm{m}) \mathrm{TE} 90^{\circ}$ elastic scattering and $0^{\circ}$ transmission simulation for the $100 \mathrm{~mm}$ sphere.

spheres the same, as the Avogadro sphere size is 100 times bigger than the $1 \mathrm{~mm}$ Si sphere.

Tunable quantum cascade (QC) lasers and methanol lasers are available in the far-IR [58-61]. Waveguiding is also possible with far-IR fibres [62-64]. Quantum dot (QD) THz detectors can be used for detection of the far-IR signal [65]. The elastically scattered signal from the Avogadro sphere can be collected by a far-IR photodetector (PD), which is connected to a digital storage oscilloscope (DSO). The $0^{\circ}$ transmitted signal can be collected by a far-IR power

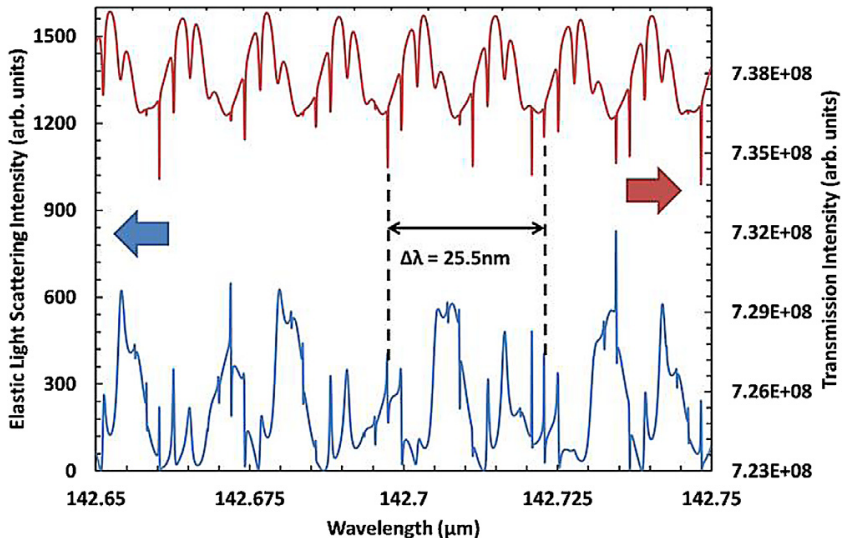

Fig. 8. Far-IR $(142 \mu \mathrm{m}) \mathrm{TM} 90^{\circ}$ elastic scattering and $0^{\circ}$ transmission simulation for the $100 \mathrm{~mm}$ sphere.

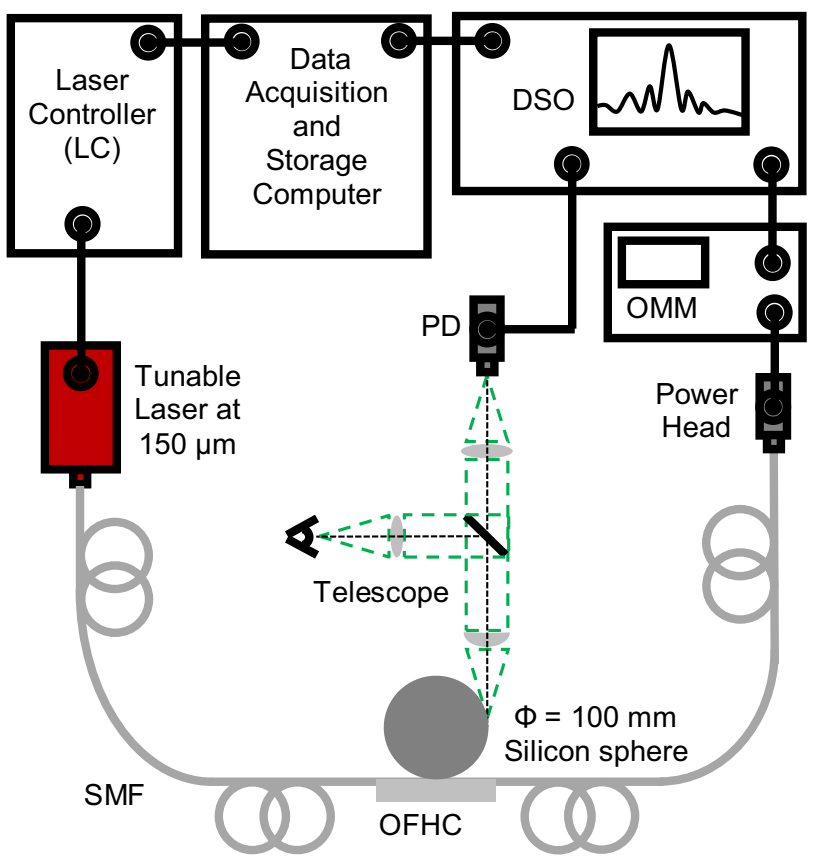

Fig. 9. Proposed far-IR $90^{\circ}$ elastic scattering and $0^{\circ}$ transmission spectroscopy setup for the Avogadro Project silicon sphere.

head (PH) of an optical multimeter (OMM). The far-IR PD and the far-IR PH can be manufactured from InGaAs heterostructure photoconductors [66].

\section{Conclusions}

Whispering gallery modes (WGMs) observed in elastic scattering from dielectric and semiconductor microspheres can provide diameter information of the sphere. Avogadro spheres are 100 times larger than the Si microspheres used in this work. Our proposal is to use elastic scattering in the far-IR, while keeping the same size parameter as the near-IR. This spectroscopic technique will provide an alternative to the optical interferometry used for diameter measurements of the Avogadro Project's Si sphere. We used elastic scattering in the near-IR for $1 \mathrm{~mm}$ Si spheres as a scale down model for the Avogadro sphere. Numerical simulations were performed using a GLMT algorithm for both the $1 \mathrm{~mm} \mathrm{Si}$ sphere and the $100 \mathrm{~mm}$ Avogadro sphere. The spectral mode spacing found using the numerical simulations agreed with the calculated and measured (for the near-IR) spectral mode spacing. The 
mass of the $1 \mathrm{~mm}$ Si microsphere was measured using a microbalance, and agreed within $3 \%$ with the mass of the microsphere estimated using the equations linking the Avogadro constant, $\mathrm{N}_{\mathrm{A}}$, with molar volume to atomic volume ratio. The diameter of the $1 \mathrm{~mm}$ Si sphere was also measured using SEM, and agreed within $0.5 \%$ with the value found using the spectral mode spacing formula Eq. (7). The GLMT simulation for the Avogadro sphere was performed using a $150 \mu \mathrm{m}$ wavelength far-IR excitation source to keep the size parameter the same as the near-IR excitation source at $1.5 \mu \mathrm{m}$. Based on the numerical simulations and the scaled down near-IR spectroscopy model, the diameter measurement of the Avogadro Project Si sphere with far-IR elastic scattering is well poised as an alternate spectroscopic method for the characterization of these roundest and purest single crystal Si spheres.

\section{Acknowledgements}

We would like to acknowledge the partial support of this work by the Scientific and Technological Research Council of Turkey (TUBITAK) project number 114F312. M.H.H., F.A., and M.R.C. would like to acknowledge support from the Higher Education Commission (HEC) of Pakistan. We would like to thank Koç University Surface Science and Technology Center (KUYTAM) for help with scanning electron microscopy (SEM) and mass measurements of the $1 \mathrm{~mm}$ Si sphere.

\section{References}

[1] Quinn TJ. The kilogram: the present state of our knowledge. IEEE Trans Instrum Meas 1991;40:81-5.

[2] Davis R. The SI unit of mass. Metrologia 2003;40:299-305.

[3] Girard G. The third periodic verification of national prototypes of the kilogram (1988-1992). Metrologia 1994;31:317-36.

[4] Mills IM, Mohr PJ, Quinn TJ, Taylor BN, Williams ER. Redefinition of the kilogram: a decision whose time has come. Metrologia 2005;42:71-80.

[5] Massa E, Nicolaus A. International determination of the Avogadro constant. Metrologia 2011;48 Foreword.

[6] Saunders JB. Ball and cylinder interferometer. J Res Nat Bur Stand 1972;76C:11-20.

[7] Sacconi A, Peuto AM, Pasin W, Panciera R, Lenaers G, Valkiers S, van den Berg M, De Bièvre P. Toward the Avogadro constant-preliminary results on the molar volume of silicon. IEEE Trans Instrum Meas 1989;38:200-5.

[8] Fujii K, Tanaka M, Nezu Y, Nakayama K, Masui R, Zosi G. Interferometric measurements of the diameters of a single-crystal silicon sphere. Rev Sci Instrum 1992;63:5320-5.

[9] Fujii K, Tanaka M, Nezu Y, Sakuma A, Leistner A, Giardini W. Absolute measurements of the densities of silicon crystals in vacuum for a determination of the Avogadro constant. In: Digest of the 1994 conference on precision electromagnetic measurements; 1994. p. 508-9.

[10] Nicolaus RA, Bönsch G. A novel interferometer for dimensional measurement of a silicon sphere. IEEE Trans Instrum Meas 1997;46:563-5.

[11] Fujii K, Tanaka M, Nezu Y, Nakayama K, Fujimoto H, De Bièvre P, Valkiers S. Determination of the Avogadro constant by accurate measurement of the molar volume of a silicon crystal. Metrologia 1999;36:455-64.

[12] Nicolaus RA, Fujii K. Primary calibration of the volume of silicon spheres. Meas Sci Technol 2006;17:2527-39.

[13] Kuramoto N, Fujii K, Yamazawa K. Volume measurements of 28Si spheres using an interferometer with a flat etalon to determine the Avogadro constant. Metrologia 2011;48:S83-95.

[14] Bartl G, Bettin H, Krystek M, Mai T, Nicolaus A, Peter A. Volume determination of the Avogadro spheres of highly enriched 28Si with a spherical Fizeau interferometer. Metrologia 2011;48:S96-S103.

[15] Azuma Y, Barat P, Bartl G, Bettin H, Borys M, Busch I, Cibik L, D’Agostino G, Fujii K, Fujimoto H, Hioki A, Krumrey M, Kuetgens U, Kuramoto N, Mana G, Massa E, Meeß R, Mizushima S, Narukawa T, Nicolaus A, Pramann A, Rabb SA, Rienitz O, Sasso C, Stock MR, Vocke RD, Waseda A, Wundrack S, Zakel S. Improved measurement results for the Avogadro constant using a ${ }^{28} \mathrm{Si}$-enriched crystal. Metrologia 2015;52:360-75.

[16] Benner RE, Barber PW, Owen JF, Chang RK. Observation of structure resonances in the fluorescence spectra from microspheres. Phys Rev Lett 1980;44:475-8.

[17] Serpengüzel A, Arnold S, Griffel G. Excitation of resonances of microspheres on an optical fiber. Opt Lett 1995;20:654-6.

[18] Yllmaz YO, Demir A, Kurt A, Serpengüzel A. Optical channel dropping with a silicon microsphere. IEEE Photon Technol Lett 2005;17:1662-4.

[19] Li X, Pyatenko A, Shimizu Y, Wang H, Koga K, Koshizaki N. Fabrication of crystalline silicon spheres by selective laser heating in liquid medium. Langmuir 2011;27:5076-80.
[20] Shi L, Fenollosa R, Tuzer TU, Meseguer F. Angle-dependent quality factor of Mie resonances in silicon-colloid-based microcavities. ACS Photon 2014:1:408-12.

[21] Davis EJ, Schweiger G. The airborne microparticle: its physics, chemistry, optics, and transport phenomena. Berlin: Springer; 2002.

[22] Zardini AA, Krieger UK, Marcolli C. White light Mie resonance spectroscopy used to measure very low vapor pressures of substances in aqueous solution. Opt Express 2006;14:6951-62.

[23] Hill SC, Rushforth CK, Benner RE, Conwell PR. Sizing dielectric spheres and cylinders by aligning measured and computed resonance locations: algorithm for multiple orders. Appl Opt 1985;24:2380-90.

[24] Psarobas IE, Leung KM. Morphology-dependent resonances in a large dielectric sphere: an asymptotic calculation using local coordinates. Phys Rev A 1992:46:2111-16.

[25] Schiller S, Byer RL. High-resolution spectroscopy of whispering gallery modes in large dielectric spheres. Opt Lett 1991;16:1138-40.

[26] Zhang J, Grischkowsky D. Whispering-gallery mode terahertz pulses. Opt Lett 2002;27:661-2.

[27] Becker P, Bièvre PD, Fujii K, Glaeser M, Inglis B, Luebbig H, Mana G. Considerations on future redefinitions of the kilogram, the mole and of other units. Metrologia 2007;44:1-14.

[28] Mills IM, Mohr PJ, Quinn TJ, Taylor BN, Williams ER. Redefinition of the kilogram, ampere, kelvin and mole: a proposed approach to implementing CIPM recommendation 1 (CI-2005). Metrologia 2006;43:227-46.

[29] Becker P. Tracing the definition of the kilogram to the Avogadro constant using a silicon single crystal. Metrologia 2003;40:366-75.

[30] Becker P, Bettin H. The Avogadro constant: determining the number of atoms in a single-crystal ${ }^{28}$ Si sphere. Phil Trans R Soc A 2011;369:3925-35.

[31] Becker P, Friedrich H, Fujii K, Giardini W, Mana G, Picard A, Pohl HJ, Riemann H, Valkiers S. The Avogadro constant determination via enriched silicon-28. Meas Sci Technol 2009;20(9):2002

[32] Zosi G. A neo-pythagorean approach towards an atomic-mass standard. Lett Nuovo Cimento 1983;38:577-80.

[33] Becker P, Bettin H, Danzebrink HU, Gläser M, Kuetgens U, Nicolaus A, Schiel D, De Bièvre P, Valkiers S, Taylor P. Determination of the Avogadro constant via the silicon route. Metrologia 2003;40:271-87.

[34] Fujii K, Waseda A, Kuramoto N, Mizushima S, Tanaka M, Valkiers S, Taylor P, Kessel R, De Bièvre P. Evaluation of the molar volume of silicon crystals for a determination of the Avogadro constant. IEEE Trans Instrum Meas 2003;52:646-51.

[35] Fujii K, Tanaka M, Nezu Y, Nakayama K, Fujimoto H, De Bièvre P, Valkiers S. Determination of the Avogadro constant by accurate measurement of the molar volume of a silicon crystal. Metrologia 1999;36:455-64.

[36] Takeda N. Spherical silicon $1 \mathrm{~mm}$ device and its clustering. In: Proc IEEE International symposium adv pack mater proc prop interfaces; 2001. p. 86-91.

[37] McKee WR. Development of the spherical silicon solar cell. IEEE Trans Comp Hybrids Manuf Technol 1982;5:336-41.

[38] Parriaux O, Gidon S, Kuznetsov AA. Distributed coupling on polished singlemode optical fibers. Appl Opt 1981:20:2420-3.

[39] Serpengüzel A, Arnold S, Griffel G, Lock JA. Enhanced coupling to microsphere resonances with optical fibers. J Opt Soc Am B 1997;14:790-5.

[40] Serpengüzel A, Demir A. Silicon microspheres for near-IR communication applications. Semicond Sci Technol 2008;23:064009.

[41] Ribeiro P, Raposo M, Eds. Photoptics 2015 (Springer, Switzerland, 2016) 174.

[42] Mohr PJ, Taylor BN, Newell DB. CODATA recommended values of the fundamental physical constants: 2010. J Phys Chem Ref Data 2012;41:043109.

[43] Mie G. Beiträge zur Optik trüber Medien, speziell kolloidaler Metallösungen. Ann Phys 1908;330:377-445.

[44] Chang RK, Barber PW. Optical effects associated with small particles. Singapore: World Scientific; 1988

[45] Chýlek P. Partial-wave resonances and the ripple structure in the Mie normalized extinction cross section. J Opt Soc Am 1976;66:285-7.

[46] Ward AD, Zhang M, Hunt O. Broadband Mie scattering from optically levitated aerosol droplets using a white LED. Opt Express 2008;16:16390-403.

[47] Murib MS, Yüce E, Gürlü O, Serpengüzel A. Polarization behavior of elastic scattering from a silicon microsphere coupled to an optical fiber. Photon Res $2014 ; 2: 45-50$

[48] Gorodetsky ML, Savchenkov AA, Ilchenko VS. Ultimate Q of optical microsphere resonators. Opt Lett 1996;21:453-5.

[49] Soltani M, Yegnanarayanan S, Adibi A. Ultra-high Q planar silicon microdisk resonators for chip-scale silicon photonics. Opt Express 2007;15(8):4694-704.

[50] Lee H, Chen T, Li J, Yang KY, Jeon S, Painter O, Vahala KJ. Chemically etched ultrahigh-Q wedge-resonator on a silicon chip. Nat Photon 2012;6(6):369-73.

[51] Dai JM, Zhang JQ, Zhang WL, Grischkowsky D. Terahertz time-domain spectroscopy characterization of the far-infrared absorption and index of refraction of high-resistivity, float-zone silicon. J Opt Soc Am B 2004;21:1379-86.

[52] Runyan WR. Silicon Semiconductor Technology. New York: McGraw-Hill; 1965.

[53] Soref R, Larenzo J. All-silicon active and passive guided-wave components for $\lambda=1.3$ and $1.6 \mu \mathrm{m}$. IEEE J Quantum Electron 1986;22:873-9.

[54] Lock JA. Improved Gaussian beam-scattering algorithm. Appl Opt 1995;34:559-70

[55] van de Hulst HC. Light scattering by small particles. New York: Dover; 1981.

[56] Nussenzveig HM. Diffraction effects in semiclassical scattering. Cambridge: Cambridge University Press; 1992. 
[57] Kužel P, Němec H, Kadlec F, Kadlec C. Gouy shift correction for highly accurate refractive index retrieval in time-domain terahertz spectroscopy. Opt Express 2010;18:15338-48.

[58] Xu L-H, Lees RM, Vasconcellos ECC, Zerbetto SC, Zink LR, Evenson KM. Methanol and the optically pumped far-infrared laser. IEEE J Quant Electron 1996;32:392-9.

[59] Knippels GMH, Yan X, MacLeod AM, Gillespie WA, Yasumoto M, Oepts D, van der Meer AFG. Generation and complete electric-field characterization of intense ultrashort tunable far-infrared laser pulses. Phys Rev Lett 1999;83:1578-81.

[60] Scalari G, Walther C, Fischer M, Terazzi R, Beere H, Ritchie D, Faist J. THz and sub-THz quantum cascade lasers. Laser Photon Rev 2009;3:45-66.
[61] Williams BS. Terahertz quantum-cascade lasers. Nat Photon 2007;1:517-25.

[62] Mendis R, Grischkowsky D. Plastic ribbon THz waveguides. J Appl Phys 2000;88:4449-51.

[63] Ung B, Mazhorova A, Dupuis A, Rozé M, Skorobogatiy M. Polymer microstructured optical fibers for terahertz wave guiding. Opt Express 2011;19:B848-61.

[64] Atakaramians S, Afshar V S, Monro TM, Abbott D. Terahertz dielectric waveguides. Adv Opt Photon 2013;5:169-215.

[65] Ueda T, Komiyama S. Novel Ultra-Sensitive Detectors in the 10-50 $\mu \mathrm{m}$ Wavelength Range. Sensors 2010;10:8411-23.

[66] Göbel T, Stanze D, Globisch B, Dietz RJB, Roehle H, Schell M. Telecom technology based continuous wave terahertz photomixing system with 105 decibel signal-to-noise ratio and 3.5 terahertz bandwidth. Opt Lett 2013;38:4197-9. 